\title{
Analyser les séances de lecture collectives à des fins de formation professionnelle
}

Analyze collective reading sessions for vocational training pruposes

Jérôme Riou

\section{OpenEdition}

\section{Journals}

\section{Édition électronique}

URL : https://journals.openedition.org/educationdidactique/5075

DOI : 10.4000/educationdidactique.5075

ISSN : $2111-4838$

\section{Éditeur}

Presses universitaires de Rennes

\section{Édition imprimée}

Date de publication : 30 décembre 2019

Pagination : $59-81$

ISBN : 978-2-7535-8041-1

ISSN : 1956-3485

Référence électronique

Jérôme Riou, "Analyser les séances de lecture collectives à des fins de formation professionnelle », Éducation et didactique [En ligne], 13-3 | 2019, mis en ligne le 03 janvier 2021, consulté le 10 octobre 2022. URL : http://journals.openedition.org/educationdidactique/5075 ; DOI : https://doi.org/10.4000/ educationdidactique.5075 


\title{
ANALYSER LES SÉANCES DE LECTURE COLLECTIVES À DES FINS DE FORMATION PROFESSIONNELLE
}

\author{
Jérôme Riou \\ Laboratoire Activité, connaissance, transmission, éducation (ACTé), \\ Université Clermont-Auvergne
}

\begin{abstract}
Dans cet article, l'auteur analyse trente-six séances de lecture collectives conduites dans les classes de cours préparatoire à la mi-novembre. Il catégorise les pratiques d'enseignement en se basant sur les proportions de durées des différents types de tâches assignées aux élèves et met en évidence des scénarios prototypiques. Il décrit ces scénarios en examinant l'articulation entre la résolution de tâches de code et la résolution de tâches de compréhension, les unités linguistiques sur lesquelles portent les traitements cognitifs des élèves, et les épisodes qui structurent les séances d'enseignement. Ainsi, il identifie des organisateurs de l'activité des professeurs et tente d'inférer les buts que ces derniers poursuivent. Il propose de construire des formations fondées sur l'analyse de séances de lecture collectives prototypiques afin que les professeurs apprennent à mieux connaitre les situations de travail auxquelles ils sont régulièrement confrontés.
\end{abstract}

Mots-clés : cours préparatoire, lecture collective, déchiffrage, compréhension, formation.

\section{Analyze collective reading sessions for vocational training pruposes}

In this article, the author analyses thirty-six collective reading sessions conducted in first grade in mid-November. It categorizes teaching practices based on the proportions of duration of the different types of tasks assigned to students and highlights prototypical scenarios. He describes these scenarios by examining the relationship between the resolution of code tasks and the resolution of comprehension tasks, the language units on which students' cognitive treatments are based, and the episodes that structure the teaching sessions. Thus, it identifies organizers of the teachers' activity and tries to infer the goals they pursue. It proposes to build training courses based on the analysis of prototype collective reading sessions so that teachers learn to better understand the work situations they are regularly confronted with.

Keywords : first-grade, collective reading, deciphering, comprehension, training. 


\section{DÉFINITION DE L'OBJET ET DE L'ENJEU}

L'enseignement du code alphabétique est habituellement conduit dans deux types de séances didactiques ${ }^{1}$. Les premières, décontextualisées et réalisées sur des unités linguistiques infralexicales (phonèmes, graphèmes, syllabes, etc.) visent le déchiffrage ${ }^{2}$ de mots ou de pseudo-mots isolés. Les secondes reposent sur l'identification de mots en contexte pour accéder à une lecture autonome de phrases ou de textes. Ce sont ces dernières que nous souhaitons étudier parce qu'elles révèlent la manière dont les maitres enseignent le décodage dans une visée d'accès à la signification de l'écrit. Nous allons donc décrire et analyser les séances au cours desquelles les élèves procèdent collectivement, sous la direction de l'enseignant, à la lecture d'un énoncé écrit (phrase ou texte) visible par tous simultanément, et qu'ils découvrent pour la première fois. Nous appellerons « séances de lecture collectives » les moments d'enseignement ainsi définis. Ceux-ci sont mis en œuvre chaque semaine dans la quasi-totalité des classes de cours préparatoire. Dans les emplois du temps que nous avons collectés, ils font partie de séances désignées par les expressions « découverte de texte», « lecture d'album », « lecture compréhension », " travail autour d'un texte ", " lecture étude du code », « lecture sens », « lecture maitrise de la langue ", "lecture à voix haute » ou encore «lecture phonologie ». Ces séances portent quelquefois le nom d'un album de lecture associé à un phonème, par exemple «Papilou et étude du son $/ \mathrm{u} / »$. Ces terminologies variées laissent entrevoir la diversité des finalités que les enseignants attribuent à la tâche de lecture et les liens qu'ils tissent entre lecture et code, lecture et compréhension ou entre lecture et oralisation.

Les séances de lecture collectives visent des apprentissages mais elles sont aussi une réponse apportée par les enseignants à l'insuffisante autonomie de décodage des élèves, à l'impossibilité de les laisser se confronter seuls à l'écrit et à l'hétérogénéité de leurs connaissances. Elles conduisent à des configurations d'activité variées et mobilisent de nombreuses composantes didactiques, ce qui rend leur description et leur analyse complexes. Par conséquent, les travaux scientifiques qui s'y sont intéressés n'ont pas encore réussi à en percer toutes les zones d'ombre.

Notre investigation devrait permettre de mieux comprendre comment les professeurs conçoivent l'articulation entre traitements cognitifs de bas niveau (le code) et de haut niveau (le sens) et dans quelle mesure ils en font la condition d'un apprentissage de la lecture réussi. L'analyse proposée aura également pour objectif de construire une typologie des pratiques d'enseignement que les formateurs d'enseignants pourraient utiliser afin de concevoir des dispositifs de formation adossés à la recherche. En effet, confrontés à une image de la diversité de leurs pratiques, les enseignants pourraient s'interroger sur les intérêts et les limites de chacune d'entre elles.

Afin de mieux connaitre les séances au cours desquelles les élèves découvrent une phrase ou un texte collectivement, nous nous intéresserons au contenu linguistique des écrits qui servent de supports à l'enseignement de la lecture et nous mettrons en évidence la proportion de graphèmes explicitement étudiés en classe. Nous nous intéresserons également à la durée de résolution des tâches prescrites aux élèves et à l'articulation de ces tâches entre elles. Nous verrons dans quelle mesure les maitres de cours préparatoire parviennent à tisser des liens entre l'enseignement du code et l'enseignement de la compréhension, et nous tenterons d'identifier des scénarios prototypiques qui pourraient s'avérer utiles pour la conception de dispositifs de formation.

\section{VERS UNE ANALYSE DES SÉANCES DE LECTURE COLLECTIVES}

\section{Bilan des recherches portant sur la conduite des séances de lecture au cours préparatoire}

Les séances de lecture de textes réalisées dans l'école française ont été étudiées par quelques rares chercheurs. En 1994, Fijalkow et Fijalkow notaient une évolution des pratiques d'enseignement de la lecture au cours préparatoire. Les enseignants déclaraient ne plus s'appuyer seulement sur la résolution de tâches de déchiffrage pour aider les élèves à identifier des mots écrits. En effet, 1/3 d'entre eux recouraient au contexte. Ils proposaient aux élèves de relire le début de la phrase ou de "sauter le mot » et de lire la suite pour tenter de le reconnaitre. Ainsi, l'activité de lecture ne semblait plus aussi centrée sur la découverte « des lettres et des syllabes » qu'elle ne l'était traditionnellement, les enseignants s'appuyant davantage sur le sens. Par ailleurs, la lecture à haute voix n'était plus seulement perçue comme une vérification des acquis mais également comme un moyen 
de communiquer oralement une information. Les auteurs concluaient toutefois que ces évolutions étaient loin d'être dominantes et regrettaient que les pratiques majoritaires s'inscrivent encore dans le prolongement des recommandations de 1923.

Dix ans plus tard, un ensemble de monographies permettait de conclure que les enseignants consacraient plus de la moitié de leur temps à l'identification et la production de mots écrits, environ un quart de leur temps à la compréhension, essentiellement de phrases, et le reste à la production de texte et à l'acculturation définie comme le « travail d'appropriation et de familiarisation avec la culture écrite " (Goigoux, 2004, p. 38). La survalorisation de l'identification et de la production de mots écrits résultait « d'une conception étapiste de l'enseignement de la lecture : les élèves devraient apprendre à identifier les mots écrits avant d'être mis face à des problèmes de compréhension de textes, maitriser les mécanismes de base avant d'accéder à la culture écrite » (Goigoux, p. 39). Même si un rééquilibrage était en train de s'opérer, l'unité linguistique « mot » demeurait une composante essentielle de l'enseignement de la lecture et constituait le principal objet d'étude et de réflexion. En 2002, Roland Goigoux dressait puis mettait à disposition de la formation un inventaire des actions et des opérations mises en ouvre par les enseignants afin de faciliter le traitement des mots écrits. Il regroupait celles qui permettent d'organiser et de gérer le travail collectif, celles qui assurent la conclusion des phases de reconnaissance des mots écrits, et celles qui guident le décodage graphophonologique. Il suggérait que les enseignants expérimentés étaient en mesure de distinguer les erreurs de traitement de l'écrit qui justifiaient une résolution publique, explicite et immédiate de celles qui pouvaient attendre car elles requéraient des compétences non disponibles pour la majorité des élèves.

Les pratiques de classe décrites en 2005 par les inspecteurs généraux et les universitaires de l'Observatoire national de la lecture réunis par Michel Fayol et Alain Bentolila semblaient assez homogènes. En se basant sur des centaines de rapports d'inspection, les auteurs du rapport sur l'apprentissage de la lecture (IGEN/ONL, 2005) indiquaient que « le modèle didactique le plus fréquemment rencontré » dans les classes de cours préparatoire reposait sur une succession de tâches proposées dans un ordre stable : discussion à partir d'une image support d'un récit ; recherche dans le texte des mots connus, la validation de la reconnaissance du mot se faisant davantage par appel au contexte que par décodage; lecture (deux ou trois fois) en continu du texte par une succession d'élèves sans véritable travail sur l'intégration syntaxique de la phrase; reprise à l'écrit (sur fiche) des mots vedettes de la leçon (en général rassemblés du fait de la présence d'un même son ou d'une même difficulté graphique), vérification de la compréhension de petites phrases extraites de la leçon par reconstitution de l'ordre des mots ou questions simples. [Rapport - no 2005-123, 2005, p. 22].

Autrement dit, les pratiques pédagogiques les plus fréquentes étaient structurées en trois étapes collectives précédant la réalisation individuelle d'exercices écrits :

- Anticipation du sens le plus souvent fondée sur l'illustration

- Identification de mots isolés

- Lecture linéaire du texte à haute voix

Les auteurs du rapport ajoutaient que ce « modèle didactique » était souvent précédé d'un travail indépendant d'étude du code basée sur la reconnaissance du son du jour (« Dans quels mots entendez-vous $[u]$ ? ») et de la graphie correspondante (« Entourez les mots où vous voyez 'ou'. »), et parfois de dictées de sons, de syllabes et de mots (IGEN/ONL, 2005, p. 22).

Les auteurs se plaçaient ensuite en posture de prescripteurs dénonçant l'insuffisance du travail d'identification des mots écrits, l'inadaptation des supports de lecture issus de la littérature de jeunesse, la pauvreté du lexique et de la syntaxe des textes étudiés, l'absence d'articulation entre identification de mots, traitement de la phrase et compréhension. En outre, ils déploraient l'abandon du travail oral sur des textes complexes tel qu'il est conduit à l'école maternelle. Bref, ils regrettaient que les supports d'apprentissage servent à la fois au travail de déchiffrage et à celui de la compréhension, ceuxci étant trop complexes pour l'un et trop simples pour l'autre. Ils dressaient donc un constat critique des pratiques de classe dont ils pointaient les incohérences, suggéraient aux enseignants de distinguer plus nettement les moments d'étude du code de ceux de la compréhension et posaient clairement la question de la différenciation des supports pédagogiques. Ces recommandations, relayées par le ministère de l'Éducation nationale, restaient cependant évasives. 
Les critiques étaient nombreuses mais les propositions étaient floues comme l'ensemble de la prescription du travail enseignant. Le projet de programme soumis au débat en 2014 et publié en 2015, après la fin de notre enquête, n'y changeait rien. Ce nouveau texte continuait simplement à faire de la construction du sens et de la reconnaissance automatique des mots écrits « deux dimensions nécessaires à la maitrise de la langue » (MEN, 2015, p. 4). Ses auteurs précisaient que la lecture collective d'un texte permet d'articuler « les processus d'identification des mots et l'accès au sens des phrases » et que ces situations de " découverte guidée » ont pour finalité la compréhension (MEN, p. 16). Seules les ressources accompagnant ces nouveaux programmes ont apporté des éclaircissements mais leur publication est postérieure à notre enquête et nous ne pouvons donc pas les retenir pour éviter un anachronisme.

En 2007, plusieurs études de cas réalisées dans le cadre de la recherche PIREF $^{3}$ coordonnée par Gérard Sensevy (2007) ont permis de comparer l'activité d'enseignants conduisant des séances de lecture à partir d'un texte commun. Le protocole imposait que ces séances se déroulent à la même période de l'année et qu'elles comportent deux passages obligés, le premier consistant à conduire un travail sur le titre du texte, le second à proposer des moments de lecture et des moments d'écriture associés. Les résultats de la première étude, qui porte sur trois classes, indiquent que la conduite des séances diffère nettement d'un enseignant à l'autre (Tauveron, 2007). L'émission d'hypothèses, par exemple, s'effectue à partir des illustrations et précède la découverte collective du texte pour l'un, intervient après la lecture de quelques phrases pour l'autre, et une fois l'intégralité du texte découvert pour le dernier. La lecture à haute voix s'effectue de manière linéaire ou fragmentée, elle est précédée ou non d'une tâche de lecture silencieuse du texte. L'identification des mots est imposée ou, au contraire, laissée au hasard des rencontres, les erreurs sont directement rectifiées par les enseignants ou les pairs dans deux classes, elles sont traitées explicitement dans la troisième. Dans cette dernière, la séance débute par un temps de déchiffrage imposé, l'objectif de l'enseignant étant que les élèves puissent oraliser le texte. Les deux autres enseignants travaillent la compréhension à des degrés divers, l'un se focalisant davantage sur les vides laissés par l'auteur, l'autre sur la signification des principales informations contenues dans les phrases, sans toutefois aborder les relations que ses informations entretiennent entre elles.

C'est également le constat établi par la seconde étude (Sensevy, 2007; 2009). Gérard Sensevy s'est attaché à comprendre, en relevant des éléments de discours, comment les enseignants permettent aux élèves de construire la signification du texte. Dans l'une des deux classes étudiées, les interactions didactiques concourent davantage que dans l'autre à développer la réflexivité des élèves et l'élaboration collective d'interprétations plausibles sur le sens du texte. Ainsi, les évaluations indiquent que les élèves de cette classe ont davantage progressé que leurs camarades en compréhension. Toutefois, l'auteur de l'étude notait qu'aucun des deux enseignants n'avait réellement pris en compte les significations noyaux du texte, autrement dit les informations permettant de construire une image mentale de la situation de lecture.

François Simon a lui aussi exploré cette problématique au moyen d'études de cas réalisées dans le cadre de sa thèse de doctorat (2004). Il a décrit les tensions auxquelles les enseignants sont confrontés lorsqu'ils doivent gérer le caractère collectif de l'activité et le caractère individuel de l'apprentissage de la lecture, des centrations sur des objectifs de traitement de l'écrit et des centrations sur des objectifs de compréhension, et enfin choisir le mode de guidage des élèves. Il a observé des pratiques qu'il juge parfois contestables. Il cite l'exemple d'une enseignante qui, s'appuyant uniquement sur la lecture silencieuse des élèves, ne parvient pas à construire collectivement la signification d'un texte. Les uns l'ont lu en intégralité alors que les autres n'ont pu déchiffrer que quelques mots. Il cite également l'exemple d'une autre enseignante qui attribue seulement une partie du texte aux élèves, celle qui lui semble la plus adaptée à leur capacité de déchiffrage. Il indique que les efforts qu'elle consentait pour différencier les activités de traitement de l'écrit s'opéraient probablement au détriment de la compréhension. En effet, le morcellement de l'énoncé ne paraissait pas de nature à favoriser la construction d'une représentation mentale de la situation.

Les orientations didactiques des enseignants aboutissaient à des pratiques contrastées. Les uns centraient leurs enseignements sur l'oralisation du texte, les autres privilégiaient le travail de compréhension et les derniers articulaient traitement de l'écrit et compréhension des unités lues. Les enseignants qui assimilaient la séance de lecture collective 
à une situation problème verbalisaient les procédures de traitement de l'écrit et les mettaient au service de la signification du texte, les autres restaient dans l'implicite et organisaient les échanges autour de significations noyaux, telles qu'elles sont définies par Sensevy (2007, 2009). Dans sa thèse, François Simon concluait que la manière dont les professionnels gèrent et régulent leur activité révèle pour partie la représentation qu'ils se font de l'apprentissage de la lecture et de son enseignement. C'est également le constat que faisaient Jaubert et Rebière (2010) dans une étude sur la « lecture-découverte » conduite dans deux classes de cours préparatoire. Les enseignantes, l'une stagiaire et l'autre expérimentée, proposaient aux élèves d'identifier des mots puis de lire le texte de façon linéaire. L'enseignante expérimentée faisait appel aux compétences syntaxiques des élèves pour traiter les erreurs d'identification de mots et les incitait à tisser des liens entre les activités de traitement de l'écrit et la production de sens lors de la tâche de lecture. En revanche, l'enseignante stagiaire verbalisait elle-même les procédures de traitement des erreurs et ne permettait pas aux élèves d'intégrer le texte lu dans la globalité de l'histoire.

Afin de faciliter le repérage et la catégorisation des activités d'enseignement/apprentissage de la lecture, Simon (2007) proposait de les situer autour des trois axes que sont la mise en projet, le traitement de l'écrit et la compréhension du texte, ces trois axes pouvant être mis en relation avec les processus cognitifs que le lecteur mobilise en situation de lecture (Giasson, 1990). Autrement dit, les séances didactiques peuvent être analysées en examinant l'activité cognitive que le professeur sollicite de la part de ses élèves. C'est pourquoi Simon regroupait sur une même représentation les traitements cognitifs mis en œuvre par les lecteurs en réponse aux sollicitations de l'enseignant.

Ses travaux s'inscrivaient dans le droit fil de ceux de Goigoux (2002) qui proposait d'analyser les séances didactiques en réponse à quatre questions : 1. Quelles sont les occasions d'apprendre que l'enseignant rend possibles à travers la conception et la réalisation de la tâche? 2. Quelles sont les activités cognitives que l'enseignant sollicite de la part des élèves? 3. Quelles sont les situations que les élèves investissent et que peuvent-ils y apprendre? 4. Qu'apprennent-ils?

À notre tour, nous allons procéder à l'analyse des séances de lecture en tentant de répondre aux deux premières questions, c'est-à-dire en prêtant attention aux opérations cognitives que l'enseignant, à travers l'enchainement de tâches qu'il propose, sollicite de la part des élèves. Nos analyses porteront sur un échantillon de séances plus vaste et plus varié que ceux de Simon, Tauveron, Sensevy ou Jaubert et Rebière, car nous avons pu le constituer à partir de classes jugées plus ou moins efficaces au terme de l'enquête Lire et Écrire (Goigoux (dir.), 2016). En cohérence avec nos questions de recherche, nous fonderons notre analyse sur un découpage original basé sur les unités linguistiques mobilisées par les tâches. Ainsi, nous saurons si les traitements cognitifs sollicités portent sur le niveau infralexical, lexical, phrastique ou textuel.

Notre analyse portera sur les leçons de lecture conduites à la mi-novembre, 10 semaines après la rentrée des classes (observations de la semaine 47 en 2013). Nous étudierons le contenu linguistique des textes qui ont servi de supports d'apprentissage et nous examinerons dans quelle mesure les choix pédagogiques des enseignants sont cohérents ou, tout du moins, influencés par les caractéristiques de ces textes supports.

Nous décrirons les scénarios didactiques mis en œuvre par les enseignants afin d'identifier les principaux organisateurs de leur activité. Nous pourrons ainsi connaitre la nature des tâches de décodage qu'ils proposent à leurs élèves, identifier la présence de tâches de reconnaissance de mots entiers n'impliquant pas d'activité explicite de déchiffrage et savoir autour de quelles unités linguistiques ils structurent leur enseignement (mot, phrase ou texte). Nous découvrirons comment ils redéfinissent leur travail au-delà de la prescription institutionnelle et nous pourrons inférer les buts qu'ils poursuivent.

\section{Questions de recherche}

Les programmes scolaires en vigueur en 2013 ne donnaient pas d'indications précises sur la mise en ouvre des séances de lecture collectives. Les enseignants les concevaient donc eux-mêmes en s'appuyant sur des concepts " organisateurs » ou « pragmatiques » (Pastré, 1999; Vidal-Gomel et Rogalski, 2007), sur des invariants opératoires (Vergnaud, 2007) que nous allons tenter d'identifier afin de faire de ces séances un objet formation. En redéfinissant la tâche prescrite, les enseignants prenaient en compte leurs caractéristiques propres, 
celles de leurs élèves et de l'institution scolaire, dans le cadre d'une activité multifinalisée orientée vers les élèves mais aussi vers eux-mêmes et vers les autres acteurs de la sphère éducative (Goigoux, 2007). Pour décrire le résultat de cette redéfinition, compromis entre deux finalités, les apprentissages enfantins et l'accomplissement personnel des maitres (Leplat, 1997), nous n'avons pas pu accéder aux commentaires des enseignants qui ont conduit les séances de lecture collectives car ils étaient disséminés sur tout le territoire national. Nous avons dû nous contenter d'analyser leurs actions filmées pour tenter d'identifier les organisateurs de leur activité et d'inférer les buts qu'ils se donnaient à eux-mêmes.

Nous nous demandons comment les maitres de cours préparatoire guident leurs élèves afin que ces derniers résolvent collectivement une tâche de lecture. Nous souhaitons savoir s'ils font de la résolution des tâches de code un préalable à la lecture à haute voix ou à l'accès au sens du texte. Nous voulons savoir quelle proportion de temps ils y consacrent, quels types de tâches ils prescrivent et comment ils les articulent aux autres composantes didactiques. Nous voulons également savoir s'ils proposent de résoudre successivement des tâches de code, de lecture et de compréhension, autrement dit s'ils font des allers-retours entre le code et le sens, et avec quelle fréquence.

Nous tenterons donc de répondre à trois questions principales :

- quelle est la nature et la durée des tâches que les enseignants proposent à leurs élèves au cours des séances de lecture collectives?

- lors de ces séances, comment articulent-ils les traitements de bas niveau et la construction de significations?

- quelle est l'unité linguistique autour de laquelle ils structurent prioritairement leur enseignement?

\section{MÉTHODOLOGIE}

Nous étudierons les séances de lecture collectives de la première semaine d'enquête de la recherche Lire et Écrire, celles du mois de novembre ( $10^{\mathrm{e}}$ semaine depuis la rentrée des classes). Nous considérons en effet que les enseignements conduits au début de l'année sont révélateurs des principales options didactiques retenues par les maitres (NRP, 2000; INSERM, 2007).
Pour mener à bien cette étude, nous disposons de multiples indicateurs nous permettant de caractériser les pratiques d'enseignement à cette période de l'année et par conséquent de contextualiser nos observations. Nous savons en particulier ce qui a été préalablement enseigné aux élèves : pour chacune des classes nous avons dressé la liste des correspondances graphophonémiques explicitement étudiées et nous avons calculé la vitesse d'enseignement de ces correspondances (tempo) ainsi que la part déchiffrable des textes proposés aux élèves lors des séances de lecture collectives (rendement effectif).

\section{La tâche, un instrument de pilotage de l'activité}

À la suite de Maurice (1996a, 1996b), nous considérons les tâches assignées aux élèves comme un instrument de pilotage de l'activité des maitres expérimentés. Ces derniers sont capables de prévoir les performances des élèves selon les tâches qu'ils leur proposent de résoudre (1996c, 2005). Ils peuvent aussi prévoir le temps nécessaire à la réalisation de ces tâches et par conséquent anticiper le déroulement des séances de classe. Nous pensons donc que le temps consacré aux différentes tâches et l'ordre dans lequel elles sont introduites sont révélateurs des choix didactiques des maitres. Nous pensons également que la manière dont ces derniers articulent ces composantes didactiques traduit leur volonté de rendre explicites les liens qui les unissent.

Nous allons donc calculer les durées de chacune des tâches qui composent les séances de lecture collectives puis nous les rapporterons à la durée totale de la séance afin de comparer d'une classe à l'autre les proportions ainsi obtenues. Nous nous appuierons sur ces données pour établir une première classification des séances. Puis nous utiliserons les suites de tâches assignées aux élèves pour construire les scénarios didactiques des enseignants et étudier la manière dont ils enchainent les tâches de différentes natures. Nous espérons ainsi pouvoir identifier les principaux organisateurs de leur activité et les buts qu'ils poursuivent.

\section{Choix des classes et des séances étudiées}

Nous avons centré nos recherches sur les classes qui étaient susceptibles de présenter les plus forts 
contrastes en code. Nous nous sommes référé aux travaux réalisés par Annette Jarlégan (2015) pour l'étude Lire et Écrire. Notre collègue a révélé des différences d'efficacité à niveau initial donné et à caractéristiques des élèves données, ce qui lui a permis de ranger par ordre d'efficacité les 131 classes de l'étude. Prenant appui sur la liste ordonnée ainsi constituée, nous avons retenu les 15 classes les plus efficaces en code, les 15 classes les moins efficaces en code et les 15 classes intermédiaires situées autour de la médiane de l'échantillon. Nous avons ainsi optimisé la diversité des profils des enseignants retenus. Nous nous sommes également donné les moyens d'identifier d'éventuels traits communs aux classes les plus ou les moins efficaces.

En visionnant les vidéos, nous nous sommes assuré que les élèves résolvaient collectivement une tâche de lecture et qu'ils rencontraient pour la première fois la phrase ou le texte. Nous avons également vérifié que les séances mettaient en scène les supports de lecture pour lesquels nous avions établi les rendements effectifs. Certaines vidéos étant inexploitables ou absentes de la base de données collective, nous avons recodé 36 séances sur 45.11 ont été conduites par des enseignants aux pratiques les plus efficaces en code, 13 l'ont été par les enseignants aux pratiques d'efficacité intermédiaire et 12 par les enseignants aux pratiques les moins efficaces en code.

\section{Codage des séances de lecture collectives}

Nous avons choisi d'identifier les opportunités d'apprendre que les enseignants offrent aux élèves. Pour cela, nous avons relevé l'ensemble des tâches prescrites pendant les séances de lecture collectives et nous avons noté leur durée.

Nous avons commencé à coder dès le début de la séance, avant même l'affichage du texte, dès les premières tâches qui concourent à sa compréhension ou à sa lecture : par exemple, le rappel des épisodes antérieurs d'un récit long, le commentaire des illustrations affichées ou l'émission d'hypothèses préalables à la lecture proprement dite. En revanche, nous n'avons pas retenu les tâches écrites individuelles réalisées à la suite de la découverte collective du texte, qui entretiennent selon les cas un lien plus ou moins étroit avec ce dernier. Nous avons interrompu la vidéo à chaque changement de consigne et nous sommes revenu en arrière pour noter soigneu- sement le temps indiqué par notre chronomètre. Nous avons relevé l'intégralité des tâches prescrites avec une précision de l'ordre de quelques secondes.

\section{Établir une typologie des séances de lecture collectives}

Pour esquisser une catégorisation des pratiques d'enseignement, nous avons eu recours à une analyse factorielle suivie d'une classification.

Nous avons choisi les variables qui nous semblaient pertinentes pour caractériser les relations entre le code et le sens et identifier les options didactiques des enseignants. Nous avons retenu 12 tâches parmi celles de la typologie de la recherche Lire et Écrire (cf. annexe I) et nous les avons regroupées pour aboutir à six variables permettant de décrire le plus simplement possible les interactions code-sens : étude de la phonographie, déchiffrage, reconnaissance de mots écrits, lecture à haute voix ${ }^{4}$, compréhension antérieure à la lecture (C-ante), compréhension en cours de lecture (C-in).

Tableau 1 : Liste des 6 ensembles de tâches

\begin{tabular}{|c|c|c|}
\hline $\begin{array}{l}\text { Variables } \\
\text { didactiques }\end{array}$ & Code & Rubrique \\
\hline \multirow[t]{2}{*}{ Phonographie } & pg4 & $\begin{array}{l}\text { Étudier les correspondances } \\
\text { entre les graphèmes et les } \\
\text { phonèmes CGP }\end{array}$ \\
\hline & pg5 & $\begin{array}{l}\text { Étudier la combinatoire ou } \\
\text { travailler sur les syllabes }\end{array}$ \\
\hline Déchiffrage & 13 & Déchiffrer un mot \\
\hline $\begin{array}{l}\text { Reconnais- } \\
\text { sance de mots } \\
\text { écrits (RME) }\end{array}$ & 12 & Reconnaitre un mot entier \\
\hline \multirow[t]{2}{*}{ Haute voix } & 14 & $\begin{array}{l}\text { Lire à haute voix une phrase ou } \\
\text { un texte }\end{array}$ \\
\hline & 15 & $\begin{array}{l}\text { Écouter la maitresse/le maitre } \\
\text { lire à haute voix une phrase ou } \\
\text { un texte }\end{array}$ \\
\hline \multirow{3}{*}{$\begin{array}{l}\text { Compréhen- } \\
\text { sion de type } \\
\text { «C-ante » }\end{array}$} & c2 & $\begin{array}{l}\text { Anticiper, formuler ou vérifier } \\
\text { des hypothèses }\end{array}$ \\
\hline & c3 & $\begin{array}{l}\text { Décrire, commenter } \\
\text { une illustration }\end{array}$ \\
\hline & $c 5$ & $\begin{array}{l}\text { Produire un rappel de récit } \\
\text { (complet ou partiel) ou un } \\
\text { rappel de texte explicatif ou de } \\
\text { consigne }\end{array}$ \\
\hline
\end{tabular}




\begin{tabular}{|l|c|l|}
$\begin{array}{l}\text { Compréhen- } \\
\text { sion de type } \\
\text { « C-in » }\end{array}$ & c4 & $\begin{array}{l}\text { Expliquer ou reformuler le sens } \\
\text { ou évoquer une représentation } \\
\text { mentale d'une phrase ou d'un } \\
\text { texte }\end{array}$ \\
\cline { 2 - 3 } & c6 & $\begin{array}{l}\text { Rendre explicite une information } \\
\text { implicite }\end{array}$ \\
\cline { 2 - 3 } & c7 & $\begin{array}{l}\text { Proposer, débattre ou négocier } \\
\text { une interprétation/des } \\
\text { interprétations }\end{array}$ \\
\hline
\end{tabular}

Comme l'indique le tableau ci-dessus, nous avons scindé les tâches de compréhension en deux sousensembles en regroupant, d'une part, celles qui précèdent la découverte collective du texte et, d'autre part, celles qui accompagnent ou suivent cette découverte. Le premier ensemble, que nous avons nommé "C-ante ", regroupe les tâches qui consistent à décrire des illustrations (c2), émettre des hypothèses (c3) ou effectuer un rappel de récit (c5). Le second, que nous avons nommé "C-in», regroupe les tâches qui consistent à expliquer, reformuler le sens du texte (c4), rendre explicite une information implicite (c6) ou proposer, débattre, négocier une ou des interprétations (c7). Nous avons également constitué un ensemble phonographie avec l'étude des correspondances graphopho- némiques (pg4) et le travail sur les syllabes ou la combinatoire (pg5), et un ensemble lecture à haute voix faite par les élèves (14) ou par l'enseignant (15). Nous avons enfin utilisé comme variables les tâches de déchiffrage (13) et celles de reconnaissance de mots écrits (12).

Nous avons calculé, pour chacune des 36 séances recodées, les proportions de durées des 6 variables didactiques précédemment définies. Puis, nous les avons introduites dans le modèle statistique afin de catégoriser les pratiques d'enseignement. Certaines d'entre elles se révèlent significativement plus longues ou significativement plus courtes que la moyenne et permettent de caractériser les cinq catégories que nous avons identifiées.

Dans la première colonne du tableau ci-dessous, les cinq catégories de séances sont ordonnées par ordre croissant de durée consacrée à l'étude du code (la $\mathrm{n}^{\circ} 1$ est celle qui consacre la plus faible proportion de temps à cette étude). Au sein de chaque catégorie, nous avons classé les séances de la plus typique à la moins typique, autrement dit de celle qui est la plus proche du parangon statistique ( centre de gravité ») de son groupe à celle qui s'en éloigne le plus (cf. tableau 2, colonne 2).

Tableau 2 : Typologie des séances de lecture collectives, parangons statistiques (en gras)

et variables didactiques caractérisant les pratiques d'enseignement

\begin{tabular}{|c|c|c|c|c|}
\hline Catégories & $\begin{array}{c}\text { Identifiants des classes } \\
\text { (en gras, les parangons } \\
\text { statistiques) }\end{array}$ & Significativement plus de & Significativement moins de & Nombre de classes \\
\hline $\mathrm{N}^{\circ} 1$ & $48,102,52,88,128,92$ & $\begin{array}{l}\text { Haute voix } \\
\text { C-ante }\end{array}$ & Déchiffrage & 6 \\
\hline No 2 & $\begin{array}{l}20,49,9,66,105 \\
112,22,34,99,72\end{array}$ & $\begin{array}{c}\text { Reconnaissance de } \\
\text { mots écrits } \\
\text { C-ante }\end{array}$ & $\begin{array}{l}\text { Haute voix } \\
\text { C-in }\end{array}$ & 10 \\
\hline $\mathrm{N}^{\circ} 3$ & $\begin{array}{c}113,80,58,127 \\
115\end{array}$ & C-in & C-ante & 5 \\
\hline $\mathrm{N}^{\mathrm{O}} 4$ & $\begin{array}{l}89,94,13,50,77,51 \\
95,125,42,63,82,98\end{array}$ & Déchiffrage & & 12 \\
\hline $\mathrm{N}^{\circ} 5$ & $60,117,118$ & Phonographie & & 3 \\
\hline
\end{tabular}




\section{Établir les scénarios didactiques} des séances de lecture collectives

Après avoir construit ces cinq catégories, nous avons réalisé des représentations graphiques des scénarios didactiques des 36 séances recodées de manière à visualiser l'ensemble des organisateurs de l'activité des enseignants et inférer les buts qu'ils poursuivent. Nous avons tracé quatre niveaux de traitement cognitif (des unités linguistiques infralexicales, en bas, à l'unité texte, en haut) sur l'axe des ordonnées et la durée en minute des tâches qui constituent la séance de lecture collective sur l'axe des abscisses (cf. figure 1). Nous avons également indiqué le code des tâches observées afin de pouvoir identifier rapidement leur nature. En voici un exemple. desquels se structurent les séances de lecture collectives et observer la fréquence des alternances de tâches de différentes natures. Ce faisant, nous avons repéré deux scénarios, le 112 et le 42, dans lesquels le travail de compréhension de type « C-in » s'effectue à partir du texte lu à haute voix par l'enseignant et non par les élèves. L'originalité de ce choix et les interrogations qu'il peut générer en formation professionnelle des enseignants nous a conduit à les isoler dans une nouvelle catégorie, la catégorie $\mathrm{n}^{\mathrm{o}} 6$.

Pour présenter chacune de ces six catégories, nous retiendrons la séance la plus typique de chacune d'entre elles, c'est-à-dire les scénarios didactiques des classes 48, 20, 113, 89, 60 et 112 .

Figure 1 : Représentation graphique d'un extrait du scénario didactique de la séance de lecture collective de la classe 42

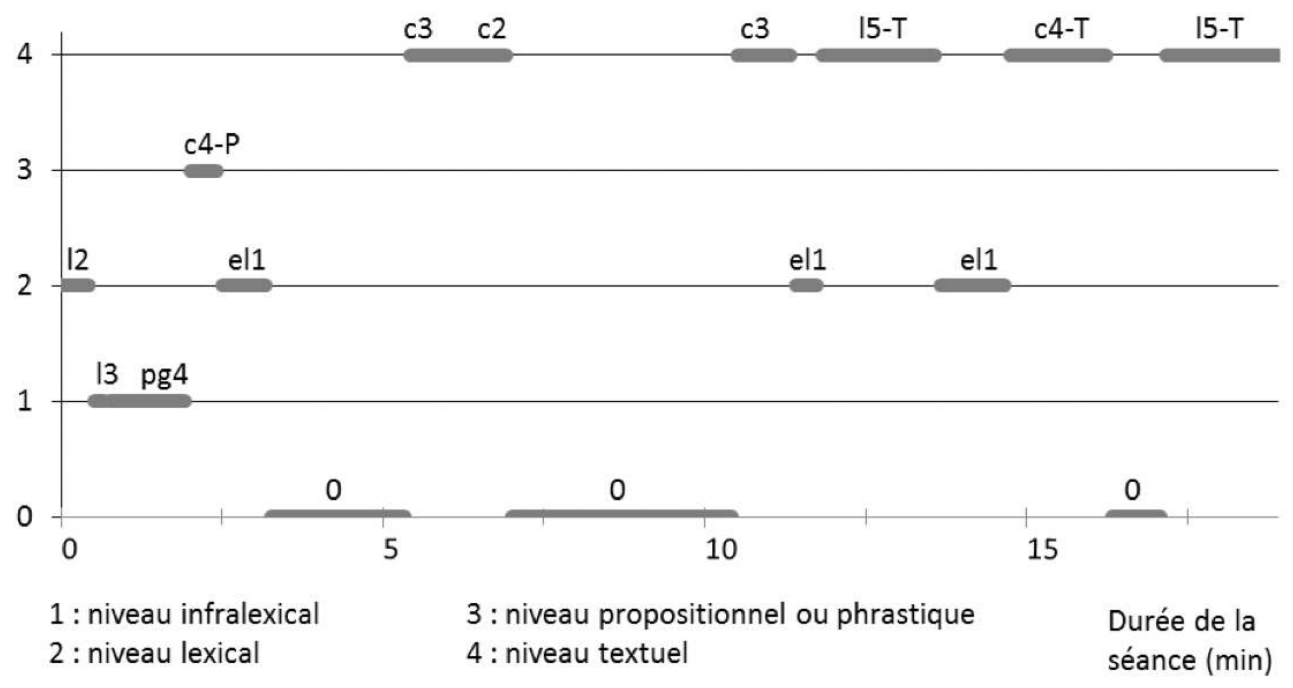

Lecture des différents types de tâche : $0:$ temps mort ${ }^{5} ; 13$ : déchiffrer un mot ; pg 4 : étudier les correspondances entre phonèmes et graphèmes ; 12 : reconnaitre un mot entier ; ell : étudier le lexique c2: anticiper, formuler ou vérifier des hypothèses ; $c 3$ : décrire, commenter une illustration ; 4 : expliquer, reformuler le sens ou évoquer une représentation mentale; 15 : écouter l'enseignant(e) lire à haute voix; $P$ : phrase; $T$ : texte

En observant l'ensemble des scénarios didactiques, nous avons pu identifier les unités linguistiques autour desquelles s'organisent majoritairement les enseignements, repérer la présence de tâches autres que celles que nous avons choisies pour élaborer nos cinq catégories, mettre au jour des épisodes répétitifs autour
Les enchainements de tâches des séances de lecture collectives

Nous avons cherché à savoir dans quelle mesure les enseignants articulaient les traitements de bas niveaux avec la construction de significations. Nous 
avons donc calculé le nombre d'enchainements de tâches de code (pg4, pg5, 13), de lecture (12, 14, 15) et de compréhension de type «C-in » (c4, c6, c7). Nous avons dénombré ces enchainements sans tenir compte de l'ordre dans lequel apparaissaient les tâches mais à la stricte condition qu'elles se suivent.

Notre premier calcul d'enchainement entre les tâches de code (pg4, pg5, 13) et les tâches de lecture (12, 14 et 15) correspond à la fréquence avec laquelle les élèves alternent les traitements cognitifs portant sur des unités linguistiques de niveau infralexical et ceux portant sur des unités linguistiques signifiantes en vue d'oraliser le texte.

Les enseignants effectuent 15 enchainements code/lecture en moyenne mais les variations sont fortes $\left(\sigma^{6}=13\right)$ entre ceux qui n'alternent jamais ces types de tâche et ceux qui le font souvent, jusqu'à 72 fois au cours d'une même séance de lecture collective (cf. tableau 3).

Tableau 3 : Enchainements des tâches de code et des tâches de lecture au cours des séances de lecture collectives

\begin{tabular}{|l|c|c|c|c|}
\hline & Moyenne & $\begin{array}{c}\text { Écart- } \\
\text { type }\end{array}$ & Minimum & Maximum \\
\hline $\begin{array}{l}\text { Nombre } \\
\text { d'enchaine- } \\
\text { ments entre } \\
\text { des tâches } \\
\text { de code et } \\
\text { des tâches de } \\
\text { lecture } \\
\mathrm{N}=36\end{array}$ & 15 & 13 & 0 & 72 \\
\hline
\end{tabular}

D'une manière générale, les enseignants qui consacrent beaucoup de temps à la résolution de tâches de code sont aussi ceux qui alternent souvent les tâches de déchiffrage et les tâches de lecture. Ainsi, ils proposent à leurs élèves des activités de traitement de l'écrit qui portent successivement sur des unités linguistiques de niveau infralexical et sur des unités linguistiques de niveau lexical ou supra-lexical. Parmi nos séances prototypiques, les enseignants des classes 48, 20 et 113 réalisent de 7 à 9 enchainements au cours de la conduite d'une séance de lecture collective, les enseignants des classes 112,89 et 60 en réalisent entre 20 et 25 .

Afin de savoir si certains d'entre eux alternent plus que d'autres les tâches de bas niveau et celles de haut niveau, nous avons calculé le nombre d'enchainements de tâches de code, de lecture et de compréhension.

Tableau 4 : Enchainements des tâches de code, de lecture et de compréhension au cours des séances de lecture collectives

\begin{tabular}{|l|c|c|c|c|}
\hline & Moyenne & $\begin{array}{c}\text { Écart- } \\
\text { type }\end{array}$ & Minimum & Maximum \\
\hline $\begin{array}{l}\text { Nombre } \\
\text { d'enchaine- } \\
\text { ments entre } \\
\text { des tâches } \\
\text { de code, de } \\
\text { lecture et de } \\
\text { compréhension } \\
\mathrm{N}=36\end{array}$ & 1 & 2 & 0 & 6 \\
\hline
\end{tabular}

Ce type d'enchainement se produit rarement, 1 fois par séance en moyenne (cf. tableau 4), et jamais dans environ la moitié des classes. Il apparait en nombre limité au cours d'une séance, d'une part, parce que les tâches de compréhension de type « C-in » sont peu nombreuses et, d'autre part, parce que ces dernières sont généralement proposées après un long moment de lecture avec ou sans déchiffrage.

En résumé, le nombre d'enchainements de tâches de code et de lecture traduit la volonté des enseignants de s'appuyer ou non sur le déchiffrage ou la phonographie pour lire le texte. Il révèle donc en partie leur mode opératoire. En revanche, le nombre d'enchainements de tâches de code, de lecture et de compréhension de type « C-in » est trop faible et trop peu discriminant pour permettre de caractériser les séances de lecture collectives.

\section{RÉSULTATS}

Dans les paragraphes qui suivent, nous analyserons les scénarios didactiques des six séances prototypiques au sein de chaque catégorie, autrement dit ceux des enseignants des classes 48, 20,113, 112, 89 et 60 . Nous les décrirons sur la base des durées allouées aux différents ensembles de tâches, des enchainements code/lecture et code/lecture/sens, et des unités linguistiques qui organisent l'activité des enseignants. Nous prendrons en compte les caractéristiques des planifications de l'étude du code, notamment le nombre de correspondances graphophonémiques explicitement 
enseignées et l'autonomie de déchiffrage de texte offerte aux élèves. Nous prêterons une attention particulière aux épisodes singuliers ou répétitifs qui structurent les scénarios didactiques.

Une première analyse fondée sur les proportions de durées

\section{La durée des séances de lecture collectives}

Avant de calculer les proportions de durée allouées aux différentes tâches qui composent les séances de lecture collectives, il nous parait souhaitable de donner un aperçu de la moyenne et de la dispersion du temps de ces dernières (cf. tableau 5).

Tableau 5 : Moyenne et dispersion du temps consacré aux séances de lecture collectives

\begin{tabular}{|l|c|c|l|l|}
\hline & Moyenne & $\begin{array}{c}\text { Écart- } \\
\text { type }\end{array}$ & Minimum & Maximum \\
\hline $\begin{array}{l}\text { Durée des } \\
\text { séances de } \\
\text { lecture } \\
\text { collectives }\end{array}$ & $\begin{array}{c}30 \mathrm{~min} \\
50\end{array}$ & $\begin{array}{c}14 \mathrm{~min} \\
59\end{array}$ & $6 \mathrm{~min} 37$ & $58 \mathrm{~min} 24$ \\
\hline $\begin{array}{l}\text { Durée } \\
\text { allouée à } \\
\text { l'enseigne- } \\
\text { ment hors } \\
\text { «temps } \\
\text { morts » }\end{array}$ & $\begin{array}{c}28 \mathrm{~min} \\
\mathrm{~N}=36\end{array}$ & $\begin{array}{c}13 \mathrm{~min} \\
55\end{array}$ & 4 min 34 & 53 min 07 \\
\hline
\end{tabular}

La durée moyenne d'une séance de lecture collective est d'environ 31 minutes $(\sigma=14 \mathrm{~min} 59)$. La séance la plus courte dure un peu moins de 7 minutes, la plus longue un peu plus de 58 minutes. Lorsqu'on soustrait les temps dévolus au fonctionnement de classe qui ont été notés comme des «temps morts » (2 minutes 14 en moyenne), la durée moyenne passe à 28 minutes $36(\sigma=13 \min 55)$.

C'est sur la base du temps que les élèves passent à résoudre des tâches, autrement dit hors « temps morts ", que nous avons calculé les proportions de durée des différentes composantes didactiques des séances de lecture collectives pour chacune des classes étudiées.

Sur la figure ci-dessous sont représentées, dans un ordre croissant de durée, les 36 séances que nous avons analysées.

Cinq des six séances prototypiques ont une durée proche, supérieure à la moyenne de l'échantillon. Une seule, la n ${ }^{\circ} 113$, est très brève. Nous en tiendrons compte dans notre analyse ultérieure.

\section{La composition des séances de lecture collectives}

Les séances de lecture collectives se composent des 6 ensembles de tâches que nous avons décrits plus haut, c'est-à-dire la phonographie, le déchiffrage, la reconnaissance de mots écrits, la lecture à haute voix, la compréhension antérieure à la lecture

Figure 2 : Durées des séances de lecture collectives, hors « temps morts », pour chacune des classes étudiées

Durées des séances de lecture collective

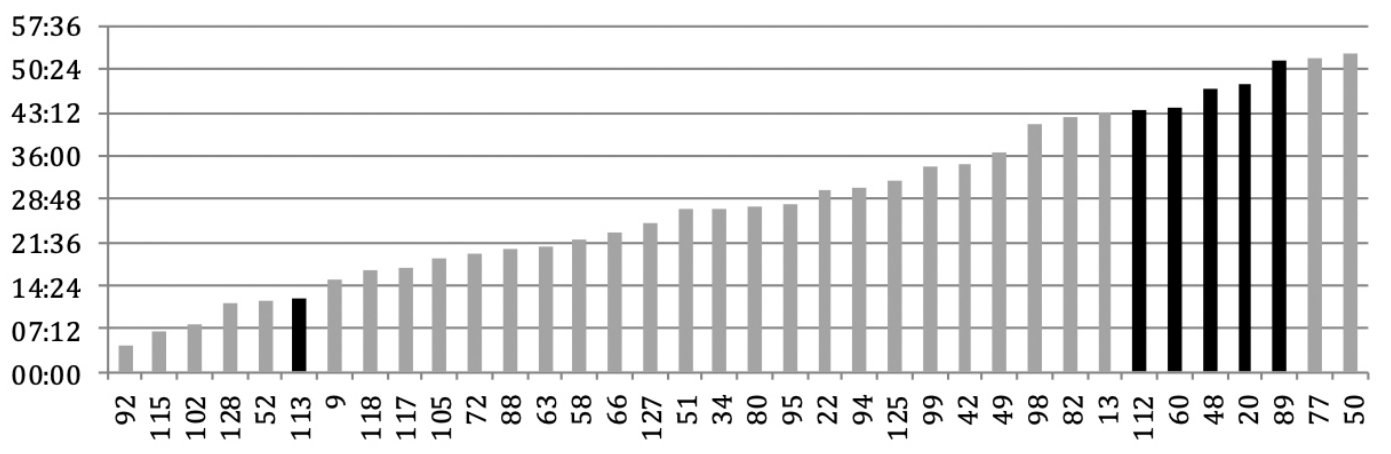

Lecture : les scénarios prototypiques apparaissent en noir. 
(C-ante) et la compréhension en cours de lecture (C-in).

Dans le tableau ci-dessous, nous avons ajouté les tâches de lecture silencieuse (11), de lexique (ell), de syntaxe (el2), et nous avons négligé toutes les autres tâches dont les proportions de durées étaient inférieures à $1 \%$ de l'ensemble. C'est pourquoi le total n'atteint pas $100 \%$.

Tableau 6 : Proportions de durée pour chaque ensemble de tâches et types de tâche dans les 36 classes étudiées

\begin{tabular}{|c|c|c|c|}
\hline \multirow{2}{*}{\begin{tabular}{l}
\multicolumn{1}{c}{ Variables } \\
Reconnaissance de \\
mots écrits (RME)
\end{tabular}} & \multirow{2}{*}{\begin{tabular}{|c|}
$\begin{array}{c}\text { Types } \\
\text { de tâche }\end{array}$ \\
12 \\
\end{tabular}} & \multicolumn{2}{|c|}{$\begin{array}{c}\text { Proportions de durée } \\
\text { en } \%\end{array}$} \\
\hline & & 11,4 & 11,4 \\
\hline \multirow{2}{*}{ Haute voix } & 14 & 20,7 & \multirow{2}{*}{26,5} \\
\hline & 15 & 5,8 & \\
\hline \multirow{3}{*}{ C-ante } & c2 & 4,3 & \multirow{3}{*}{15,9} \\
\hline & c3 & 6,0 & \\
\hline & c5 & 5,6 & \\
\hline \multirow{3}{*}{ C-in } & $c 4$ & 5,9 & \multirow{3}{*}{6,9} \\
\hline & c6 & 0,9 & \\
\hline & c7 & 0,1 & \\
\hline \multirow{2}{*}{ Phonographie } & $\mathrm{pg} 4$ & 3,2 & \multirow{2}{*}{4,1} \\
\hline & pg5 & 0,9 & \\
\hline Déchiffrage & 13 & 16,4 & 16,4 \\
\hline Lecture silencieuse & 11 & 6,7 & 6,7 \\
\hline Lexique & ell & 4,1 & 4,1 \\
\hline \multirow[t]{2}{*}{ Syntaxe } & el2 & 5,9 & 5,9 \\
\hline & & Total : & 97,9 \\
\hline
\end{tabular}

La lecture à haute voix occupe en moyenne un peu plus d'un quart de la durée d'une séance, le déchiffrage ou la compréhension qui précèdent la lecture du texte ( $\mathrm{C}$-ante) environ un sixième. La compréhension de type « C-in », le lexique et la syntaxe occupent chacun moins de $7 \%$ du temps. Nous constatons donc que le travail de compréhension s'effectue essentiellement en amont de la découverte collective du texte.

À l'intérieur des ensembles composés de plusieurs tâches, la contribution de chaque tâche est inégale. Dans l'ensemble "Haute voix ", il y a 4 fois plus de lecture effectuée par les élèves (14) que par les enseignants (15). Dans l'ensemble " $C$-in », le travail de compréhension consiste essentiellement à expli- quer, reformuler le sens ou évoquer une représentation mentale (c4), les deux autres tâches sont en quantité négligeable. Enfin, dans l'ensemble «Phonographie », la proportion de durée qui porte sur la mise en correspondance des graphèmes et des phonèmes (pg4) est 3 fois plus élevée que celle du travail sur les syllabes ou la combinatoire (pg5).

Les écarts-types élevés témoignent de la grande diversité des proportions de durée allouées aux différents ensembles de tâches (cf. tableau 7). Les valeurs minimales sont souvent égales à 0 et les valeurs maximales permettent d'apprécier l'amplitude des intervalles.

Tableau 7 : Moyennes et dispersion des proportions de durée consacrées aux ensembles de tâches utilisés comme indicateurs dans notre analyse factorielle

\begin{tabular}{|l|c|c|c|c|c|c|}
\hline & $\begin{array}{c}\text { Phono- } \\
\text { graphie }\end{array}$ & $\begin{array}{c}\text { Déchif- } \\
\text { frage }\end{array}$ & RME & $\begin{array}{c}\text { Haute } \\
\text { voix }\end{array}$ & $\begin{array}{c}\text { C- } \\
\text { ante }\end{array}$ & C-in \\
\hline moyenne & 4,1 & 16,4 & 11,4 & 26,5 & 15,9 & 6,9 \\
\hline $\begin{array}{l}\text { écart- } \\
\text { type }\end{array}$ & 8,3 & 12,3 & 14,3 & 16,8 & 11,4 & 6,9 \\
\hline min & 0 & 0 & 0 & 4,8 & 0 & 0 \\
\hline $\max$ & 37,7 & 51,2 & 53,1 & 72,8 & 44,8 & 27,6 \\
\hline
\end{tabular}

\section{La composition des 6 séances prototypiques}

Sur la figure 3 ci-dessous sont représentées les proportions de durées allouées aux différentes composantes didactiques de chacun des scénarios prototypiques que nous avons retenus. Ces scénarios sont rangés selon la part attribuée à l'enseignement du code (pg4, pg5 et 13), dans un ordre croissant.

Lecture : les scénarios sont rangés par ordre croissant de proportions de durées allouées au code.

Cette représentation graphique permet tout d'abord d'identifier quelques contrastes forts entre les séances. Ainsi, l'enseignante de la classe 60 consacre $45 \%$ du temps de la séance à l'étude du code alors que l'enseignante de la classe 48 y consacre moins de $5 \%$. La reconnaissance de mots écrits occupe $35 \%$ du temps de la séance de lecture collective de la classe 20, c'est une part nettement plus importante que celle des cinq autres classes. En revanche, 
Figure 3 : Répartition des durées exprimées en pourcentage pour chacune des 6 séances prototypiques

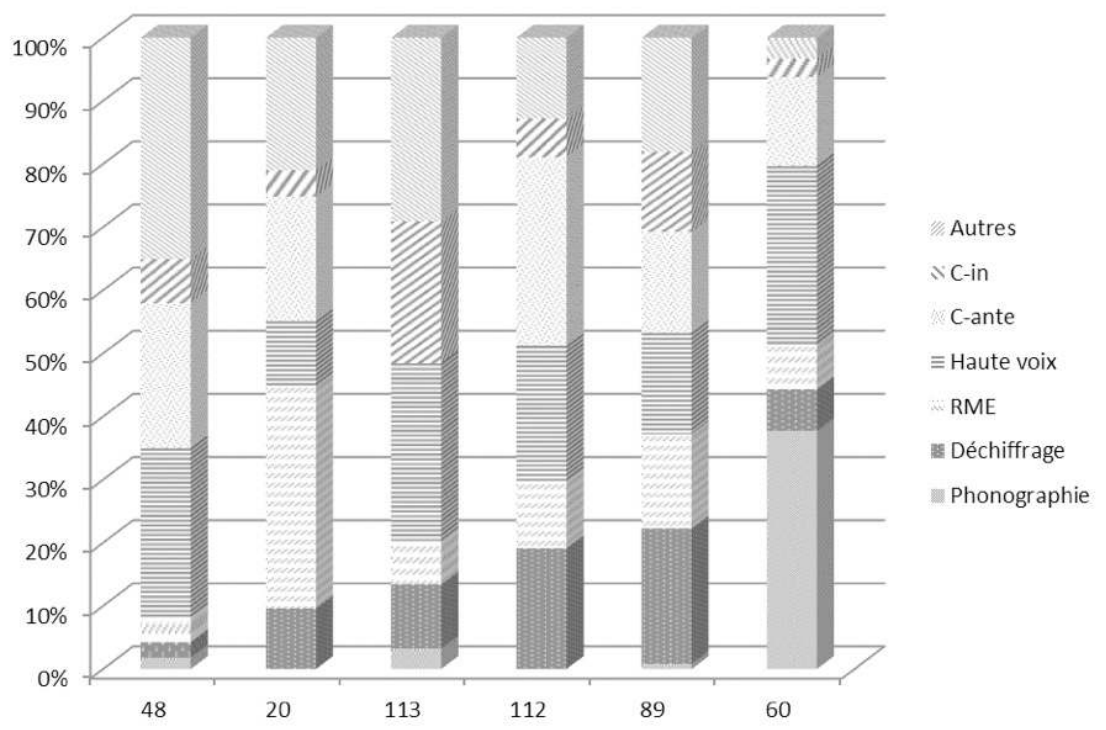

comme dans la classe 112, l'enseignante n'a proposé aucune tâche de phonographie.

Contrairement à ses collègues (mais n'oublions pas que la séance analysée est très brève), l'enseignante de la classe 113 ne propose aucune tâche de compréhension précédant la découverte collective du texte (C-ante) mais accorde une proportion de durée significativement plus importante que les autres à la compréhension qui suit cette découverte (C-in).

Poursuivons et affinons l'analyse en examinant les séances une à une sans perdre de vue que chacune révèle les principales caractéristiques des séances de sa catégorie.

\section{Analyse des séances prototypiques}

\section{La séance de lecture collective de la classe 48}

L'enseignante de la classe 48 utilise le manuel de lecture Ribambelle. Elle propose aux élèves de découvrir un extrait de l'album Poulette Crevette de Françoise Guillaumond et Clément Oubrerie (cf. tableau 8). Les élèves peuvent déchiffrer seuls $24,4 \%$ des graphèmes du texte s'ils connaissent uniquement les 5 correspondances graphophonémiques étudiées depuis la rentrée des classes.
Tableau 8 : Texte support d'apprentissage de la lecture proposé à la mi-novembre par l'enseignante de la classe 48

Le jour? Ils suivent Crevette partout. La nuit? Ils restent tout près d'elle. Mais Crevette ne dit rien : ni le jour, ni la nuit.

Lecture : les graphèmes explicitement enseignés apparaissent en gras.

La séance peut être découpée en 4 épisodes. Tout d'abord, l'enseignante demande aux élèves de se remémorer les précédentes lectures. Pour les y aider, elle leur montre les illustrations. Puis, afin qu'ils se construisent une image mentale de la situation, elle les incite à faire des liens avec leur propre vécu. Elle leur propose ensuite de lire à haute voix le texte déjà découvert. Elle leur laisse le temps de prendre connaissance silencieusement des deux dernières phrases avant de les oraliser. Elle attire l'attention des élèves sur le repérage du début et de la fin de chacune d'elles à l'aide des majuscules et des points. Enfin, elle les laisse découvrir le nouveau texte silencieusement et formuler des hypothèses sur la suite du récit. À la fin de la séance, le texte est lu à haute voix, l'enseignante insiste à nouveau sur les aspects syntaxiques.

Dans cette séance qui dure un peu plus de 47 minutes (hors « temps morts»), seules deux courtes tâches de compréhension portent sur le texte étudié. 
L'essentiel du temps est consacré à la lecture à haute voix et à la lecture silencieuse. Les tâches de déchiffrage et de reconnaissance de mots écrits sont rares, tout comme celles de compréhension de type «C-in».

En résumé, pour la catégorie $\mathrm{n}^{\circ} 1$, la séance s'organise autour de l'unité texte et mobilise essentiellement des tâches de lecture. L'oralisation du texte semble être le principal objectif de l'enseignante qui n'essaie pas d'articuler les traitements de bas niveau et la construction de significations.

\section{La séance de lecture collective de la classe 20}

L'enseignante de la classe 20 utilise le manuel Que d'histoire!. Le texte qu'elle propose aux élèves est extrait de l'album Le grand voyage du petit millepattes, de Françoise Guillaumond et Virginie Jamin (cf. tableau 9). Les élèves peuvent déchiffrer seuls $60 \%$ du texte s'ils connaissent les 11 correspondances graphophonémiques étudiées depuis la rentrée des classes.

Tableau 9 : Texte support d'apprentissage de la lecture proposé à la mi-novembre

par l'enseignante de la classe 20

Le grand voyage du petit mille-pattes

Alors le mille-pattes décide de patiner sur la glace.

Il avance : une patte, deux pattes, trois pattes, quatre pattes, cinq pattes,... dix pattes, .. . cent pattes, ... mille pattes! Patatrac! Il tombe. Cric et crac! Il se casse une patte.

Lecture : les graphèmes explicitement enseignés apparaissent en gras.

Au début de la séance, l'enseignante souhaite que les élèves produisent un rappel de récit. Comme ils n'y parviennent pas, elle leur propose de commenter les illustrations et d'émettre des hypothèses sur les circonstances dans lesquelles le mille-pattes s'est blessé. Par la suite, elle leur distribue le texte sur fiche et leur demande d'entourer les mots qu'ils connaissent. Au bout d'environ 6 minutes, elle les invite à travailler sur le support collectif. Elle leur propose alors de repérer la première phrase du texte et d'entourer les mots déjà rencontrés, puis de lire la phrase à haute voix. Elle procédera ainsi pour chacune des phrases du texte.

Au cours de la phase collective de reconnaissance de mots écrits, lorsqu'un élève se trompe, l'ensei- gnante rappelle le nom des lettres et la valeur des graphèmes pour le déchiffrer. Si nécessaire, elle note la proposition de l'élève au-dessus du mot pour les comparer. Toutefois, les tâches de déchiffrage sont plutôt rares, elles alternent à seulement 8 reprises avec celles de lecture. Les tâches de compréhension qui portent sur le texte découvert collectivement sont quasi-inexistantes.

Le travail de syntaxe qui jalonne la découverte collective du texte porte sur l'identification des phrases et sur le repérage, la connaissance et la fonction des signes de ponctuation. La catégorisation des mots de la phrase ou l'étude de la valeur des marques grammaticales (formes du verbe, marques de genre et de nombre) sont absentes.

En résumé pour la catégorie $\mathrm{n}^{0} 2$, l'enseignante de la classe 20 organise ses enseignements autour de l'unité linguistique phrase sans jamais demander la lecture à haute voix de l'intégralité du texte. La quasi-totalité des tâches qui portent sur la compréhension sont concentrées au début de la séance et concernent une partie du récit déjà découverte. Les traitements cognitifs des élèves sont majoritairement orientés vers la reconnaissance des mots écrits en vue de l'oralisation des phrases.

\section{La séance de lecture collective de la classe 113}

La séance de lecture collective de la classe 113 est relativement courte en comparaison des autres séances prototypiques, elle dure à peine plus de 12 minutes (hors « temps morts»). L'enseignante utilise le manuel Léo et Léa, elle a choisi d'étudier le texte Repu! (page 29) que les élèves peuvent déchiffrer seuls à hauteur de $68 \%$ s'ils connaissent les 12 correspondances graphophonémiques étudiées depuis la rentrée des classes (cf. tableau 10).

Tableau 10 : Texte support d'apprentissage de la lecture proposé à la mi-novembre par l'enseignante de la classe 113

Repu!

Milo, le chat, passe sur le mur. Il part à la chasse. Il avale six rats : repu, le chat! Il s'affale sur le lit. Puis il se lave. Léo arrive, affamé. Il avale... sa purée.

Lecture : les graphèmes explicitement enseignés apparaissent en gras $^{7}$. 
Au début de la séance, l'enseignante demande aux élèves de prendre connaissance du texte et de repérer le nom des personnages, ceux dont il est habituellement question dans le manuel. Elle leur propose de lire le titre et explique le sens du mot « repu». Ensuite, les phrases du texte sont repérées et dénombrées. Enfin, elles sont lues à haute voix. Ces lectures sont entrecoupées de tâches de compréhension ou de lexique, quelquefois de tâches de déchiffrage lorsque les mots ne sont pas entièrement reconnus. L'identification des mots s'opère alors par décomposition syllabique, l'enseignante utilisant quelquefois les gestes Borel-Maisonny pour permettre aux élèves de se remémorer la valeur des graphèmes. Lorsque ces aides sont insuffisantes, les mots sont donnés par les pairs ou par l'enseignante elle-même. La séance observée précède la résolution de tâches écrites individuelles et ne dure qu'une quinzaine de minutes.

La part déchiffrable élevée (68\%) indique que le texte est accessible aux élèves du point de vue du déchiffrage, elle offre donc à l'enseignante la possibilité de consacrer davantage de temps à l'élaboration du sens du texte. C'est le constat que nous faisons lorsque nous comparons son scénario didactique à ceux des autres séances prototypiques. Par conséquent, les tâches de code et de lecture alternent peu. En revanche, à deux reprises, on observe une succession de tâches de code, de lecture et de compréhension, autrement dit le passage de traitements dits « de bas niveau » à la construction de significations.

Il nous semble toutefois que le support de lecture utilisé, qui manque de cohérence sémantique et syntaxique, se prête davantage à un travail de déchiffrage en contexte qu'à un véritable travail de compréhension. Par ailleurs, nous avons constaté que l'enseignante ne suivait pas strictement la planification de l'étude du code proposée par le guide pédagogique. Elle a étudié la correspondance graphophonémique « y, /i/ » qui est absente du texte de lecture et n'a pas étudié les correspondances « $\mathrm{u}$, $/ \mathrm{Y} / »$ et « $\mathrm{s}, / \mathrm{s} /$ » ainsi que les graphèmes muets qui ont pourtant servi à le produire.

En résumé, la séance de la catégorie $\mathrm{n}^{0} 3$ est structurée autour de l'unité phrase comme celle de la catégorie $n^{\circ} 2$ représentée par la classe 20. En revanche, l'oralisation ne constitue pas une finalité mais un préalable au travail de compréhension. L'enseignante utilise un texte déchiffrable à presque $70 \%$ et consacre peu de temps à l'étude du code.

\section{La séance de lecture collective de la classe 112}

L'enseignante de la classe 112 utilise les albums de la collection "Libellule » éditée par Sedrap. La séance observée porte sur une phrase du récit intitulé La sorcière de la mer de Nicolas de Hirshing et Isabelle Nègre, phrase que les élèves peuvent déchiffrer seuls à hauteur de $62 \%$ s'ils connaissent les 22 correspondances graphophonémiques étudiées depuis la rentrée des classes (cf. tableau 6).

Tableau 11 : Phrase support d'apprentissage de la lecture proposée à la mi-novembre par l'enseignante de la classe 112

La sorcière de la mer

À bout de patience, le maire réunit un soir tout le village afin de trouver une solution pour se débarrasser de cette maudite sorcière.

Lecture : les graphèmes explicitement enseignés apparaissent en gras.

La séance débute par un épisode relativement long qui précède la découverte collective du texte. L'enseignante demande tout d'abord aux élèves de produire un rappel de récit puis elle lit à haute voix le texte correspondant. Sa lecture est très expressive et les élèves participent à l'énoncé des dialogues en se mettant dans la peau des personnages. À plusieurs reprises, l'enseignante s'interrompt pour préciser le sens de certains mots, de certaines expressions ou du texte lui-même. Puis, les élèves commentent une nouvelle illustration sur laquelle figure le mot « Mairie ». La valeur du graphème « ai » est identifiée à l'aide du graphème « è ». Cette mise en correspondance graphophonémique est suivie d'émission d'hypothèses. La séance a débuté depuis environ 28 minutes lorsque l'enseignante propose aux élèves de lire une nouvelle phrase au fur et à mesure qu'elle l'écrit au tableau. Elle marque des pauses pour inciter au déchiffrage de certains mots. Par exemple, elle s'arrête après chacune des syllabes du mot « réunit » et barre la lettre « $\mathrm{t} »$ qui est muette. Elle laisse quelquefois les élèves émettre des hypothèses sur la suite de l'énoncé avec l'aide du contexte littéral. Une fois l'écriture de la phrase terminée, elle leur demande de la lire sans déchiffrer les mots, de la relire en y mettant l'intonation, puis de la lire à nouveau. La séance se termine par une chasse aux mots, autre- 
ment dit par une recherche collective de mots dans le texte étudié.

La séance est donc structurée en deux épisodes très distincts. Le premier porte sur un texte lu par l'enseignante et conduit à la résolution de tâches de compréhension et de lexique, le second concerne la découverte d'une phrase et il est marqué par de très nombreuses tâches de déchiffrage. Les tâches de compréhension ne portant pas sur la phrase lue, nous n'observons aucune articulation entre code et sens.

En résumé pour la catégorie $n^{\circ} 4$, la séance s'organise d'abord autour de l'unité texte puis ensuite autour de l'unité phrase. Pendant deux tiers du temps, les élèves résolvent des tâches de compréhension qui portent sur une partie connue du récit relue par l'enseignante. Ils découvrent ensuite une nouvelle phrase en alternant fréquemment des tâches de lecture à haute voix et des tâches de déchiffrage. Il semble que l'enseignante poursuive donc un double objectif de compréhension et d'oralisation mais en deux temps distincts.

\section{La séance de lecture collective de la classe 89}

L'enseignante de la classe 89 propose de lire un extrait de l'album Drôle d'année pour Zoé! de Dominique Falda. Les élèves peuvent déchiffrer seuls $57,1 \%$ du texte s'ils connaissent les 16 correspondances graphophonémiques étudiées depuis la rentrée des classes (cf. tableau ci-dessous).

Tableau 12 : Texte support d'apprentissage de la lecture proposé à la mi-novembre par

l'enseignante de la classe 89

Ce printemps, devine ce qui m'est arrivé! Tu ne trouves pas? Tu donnes ta langue au chat? J'étais gaie comme un pinson... lorsqu'une hirondelle a déposé dans ma main une graine venue d'Afrique. Alors vite, je l'ai plantée! Une minute après, un énorme baobab trônait dans mon jardin. Et je ne te raconte pas de salades! Tu ne me crois pas? Tant pis pour toi!

Lecture : les graphèmes explicitement enseignés apparaissent en gras.

L'enseignante relit à haute voix le début de l'histoire. Elle montre les illustrations qui correspondent à la suite du récit, demande aux élèves de les commenter et d'émettre des hypothèses. Puis, elle dévoile le texte écrit au tableau dans lequel les lettres muettes sont marquées par des points. Elle propose de lire le contenu des paragraphes entourés, ceux qui se répètent tout au long du récit, et de découvrir silencieusement le reste de l'écrit. Elle demande ensuite aux élèves de reconnaitre certains mots du texte en commençant par ceux qui donnent des informations sur la compréhension, par exemple « hirondelle». Elle essaie de vérifier la validité des hypothèses émises et des commentaires produits à partir des illustrations. Ce travail de vérification s'opère tout au long de la découverte de texte. L'enseignante accepte néanmoins que les élèves proposent des mots tels que "un », "une » ou « ma », moins directement utiles au but qu'elle poursuit. Elle procède à des décompositions syllabiques et à la mise en relation de certaines correspondances graphophonémiques. Selon les cas, elle effectue un déchiffrage partiel ou complet. Plus rarement, elle propose de résoudre une tâche d'encodage afin de comparer une proposition erronée avec un mot contenu dans le texte. À la fin de la séance, elle lit lentement et distinctement ce dernier en pointant les mots avec sa règle. Les élèves ont alors pour consigne de suivre attentivement et silencieusement sa lecture pour se préparer à la lecture du soir. Enfin, elle pose plusieurs questions afin de combler les vides laissés par l'auteur.

Lors de cette séance de lecture collective, plus de la moitié des graphèmes du texte étudié est directement déchiffrable. Toutefois, l'épisode de reconnaissance de mots écrits conduit à la résolution publique de nombreuses tâches de déchiffrage, y compris lorsque les élèves formulent la bonne proposition. Les tâches de code alternent à 20 reprises avec celles de lecture. Celles de compréhension de type « C-in » sont courtes et peu fréquentes, à l'exception de l'épisode final pendant lequel l'enseignante lève plusieurs implicites du texte. Toutefois, nous observons à 4 reprises une alternance code/lecture/sens, ce qui constitue un indicateur de l'articulation entre des traitements de bas niveau et la construction de signification.

En résumé pour la catégorie $\mathrm{n}^{0} 5$, la séance se structure autour de l'unité texte dont l'enseignante vise la compréhension. Toutefois, pendant les deux tiers du temps, les tâches alternent entre reconnaissance de mots écrits et déchiffrage, ce dernier étant majoritaire pour ce qui est de la durée. 


\section{La séance de lecture collective de la classe 60}

La classe 60 est un cours double composé d'élèves de cours préparatoire et d'élèves de cours élémentaire première année. Le texte qui sert de support d'apprentissage de la lecture a été rédigé par l'enseignante après que les élèves aient assisté à un spectacle de contes. Selon l'enquêtrice qui a observé le fonctionnement de la classe pendant 3 semaines, l'écriture de textes qui portent sur des situations vécues est une pratique ordinaire de l'enseignante.

Les élèves peuvent déchiffrer seuls $37 \%$ du texte s'ils connaissent uniquement les correspondances graphophonémiques préalablement étudiées (cf. tableau 6).

\section{Tableau 13 : Texte support d'apprentissage de la lecture proposé à la mi-novembre par \\ l'enseignante de la classe 60}

Vendredi matin, la classe est allée voir un conteur dans la salle du Coudray. Le conteur nous a raconté deux contes gourmands: Bonhomme Misère et Les bonbons de la sorcière. Bonhomme Misère avait une petite maison. Il avait un petit jardin. Et il avait aussi un très beau pommier avec des pommes rouges. Tous les jours, en allant à l'école, les enfants montaient dans le pommier. Ils volaient les pommes de Bonhomme Misère. Ils cassaient des branches. Bonhomme Misère n'était pas content du tout. Mais un jour, un magicien arriva dans la petite maison de Bonhomme Misère.

Lecture : les graphèmes explicitement enseignés apparaissent en gras.

La séance débute par un rappel de la situation vécue, l'enseignante rendant explicites certaines informations nécessaires à la compréhension du spectacle de contes. Puis, les élèves de $\mathrm{CP}$ recherchent des mots dans les deux premières phrases du texte et les lisent. L'enseignante attire leur attention sur la morphologie et l'étymologie du mot « bonhomme ». Les élèves de CEl, d'abord spectateurs, se voient confier un travail de remise en ordre des paragraphes de l'histoire intitulée Bonhomme Misère. L'enseignante propose alors aux élèves de $\mathrm{CP}$ de lire le texte selon un mode linéaire. Elle leur demande de déchiffrer les mots qui ne sont pas reconnus et d'autres qu'elle choisit. Elle attire leur attention sur la lettre d'attaque, les syllabes et quelques correspondances graphophonémiques propres à ces mots. Elle rejoint ensuite les élèves de CEl. Pendant ce temps, les élèves de CP colorient les digrammes du texte en respectant un code couleur: « ou » en orange, « on » en jaune, « an » et « en » en vert, «ch » en bleu. L'enseignante revient avec eux pour lire le texte à haute voix et émettre des hypothèses sur la suite du récit.

La réalisation des tâches de code occupe près de $45 \%$ de la durée de la séance. Pour l'essentiel, il s'agit d'identifier des correspondances entre les graphèmes et les phonèmes. Toutefois, de nombreuses tâches de déchiffrage accompagnent l'oralisation du texte, les alternances entre ces tâches et celles de lecture sont donc nombreuses. En revanche, l'articulation entre code et sens est absente, et pour cause, puisqu'aucune tâche de compréhension ne suit la découverte collective du texte (absence de tâches de type « C-in »).

En résumé pour la catégorie $\mathrm{n}^{0} 6$, la séance s'organise autour de l'unité linguistique texte. L'enseignante propose à ses élèves de résoudre des tâches de phonographie et de déchiffrage de mots afin de l'oraliser, ce qui constitue très probablement la finalité du scénario didactique qu'elle a conçu.

\section{Synthèse}

Nous venons de décrire les séances les plus typiques des six catégories de pratiques que nous avons identifiées, en commençant par celle qui accorde le moins de place au code et en terminant par celle qui en accorde le plus. Nous allons distinguer deux ensembles de séances.

Le premier ensemble réunit un peu plus de la moitié des scénarios didactiques étudiés, 21 très précisément. Il est composé des catégories $\mathrm{n}^{0} 1$, 2 et 3 représentées par les séances des classes 48 , 20 et 113. Les enseignants de ce premier ensemble consacrent peu de temps au déchiffrage et ne proposent que de rares alternances entre des tâches de lecture et de code (de 7 à 9 fois seulement par séance). Leurs scénarios didactiques débutent en général par une phase dédiée à la compréhension qui précède la lecture du texte ( $\mathrm{C}$-ante) et se poursuivent en consacrant un temps significatif soit à la lecture à haute voix, soit à la reconnaissance visuelle de mots entiers (accès direct au sens sans explicitation des procédures de déchiffrage). Une minorité de scénarios cependant est structurée autour des tâches de reformulation ou d'explication du sens au fil de la découverte du texte (compréhension de 
type « C-in »). Certains enseignants étudient le texte phrase après phrase, après avoir passé un temps quelquefois conséquent à localiser chacune d'entre elles dans l'espace graphique (utilisation des majuscules et des points), d'autres se soucient peu du découpage phrastique et proposent des tâches qui portent essentiellement sur le niveau textuel.

Le second ensemble, composé de 15 scénarios, réunit les catégories $\mathrm{n}^{\mathrm{0}} 4,5$ et 6 , celles qui accordent le plus d'importance à l'étude du code, autrement dit au déchiffrage et à la phonographie. Dans ces scénarios, le déchiffrage en contexte apparait comme le principal organisateur de l'activité d'enseignement, c'est pourquoi l'alternance des tâches de code et de lecture est fréquente, 20 à 25 fois au cours d'une séance. Les séances observées reposent sur une succession de tâches dont la finalité est l'oralisation (sans accès explicite à la signification) ou la compréhension de texte. Les tâches de compréhension de type «C-in » sont systématiquement précédées de tâches d'oralisation mais les proportions des durées dédiées aux tâches de déchiffrage sont très variables. À noter enfin que le scénario de la classe 112 est original dans la mesure où il s'organise en deux temps très distincts, le déchiffrage de la phrase étant précédé d'un travail de compréhension de texte lu par l'adulte.

Le tableau ci-dessous résume les principaux éléments de la synthèse. L'observation de l'importance accordée aux différentes tâches et de leur succession dans le déroulement des séances nous a permis d'inférer les principaux buts poursuivis par les enseignants, à savoir l'oralisation d'une phrase, d'un texte ou leur compréhension.
Nous pensions que la part directement déchiffrable des textes influençait la préparation et la conduite des séances de lecture collectives. En effet, le choix de textes qui contiennent une grande quantité de graphèmes déjà étudiés aurait pu conduire à un investissement moindre des tâches de code, et celui de textes qui en contiennent peu à un investissement plus conséquent. Or, ce n'est pas toujours ce que nous constatons. S'il est vrai que l'enseignante de la classe 60 accorde beaucoup de temps au code avec un texte peu déchiffrable, ce n'est pas le cas de l'enseignante de la classe 48 qui utilise pourtant un texte qui l'est encore moins. D'autres facteurs entrent en ligne de compte dans la conduite de l'activité d'enseignement, notamment les buts poursuivis par les maitres et les organisateurs sur lesquels ils s'appuient pour atteindre ces buts. Autrement dit, lorsque le texte est peu déchiffrable, soit l'enseignant consacre beaucoup de temps au déchiffrage pour enseigner ce qui fait défaut aux élèves, soit au contraire, il élude les difficultés en donnant lui-même les informations manquantes sans les expliciter.

\section{DISCUSSION}

Nous avons étudié les séances au cours desquelles les élèves résolvent collectivement une tâche de lecture à partir d'un support d'apprentissage commun, séances que les auteurs du rapport IGEN/ONL (2005) résumaient en trois phases quasi immuables dans toutes les classes de cours préparatoire : anticipation du sens le plus souvent fondée sur l'illustration, identification de mots isolés en privilé-

Tableau 14 : Synthèse des buts et des organisateurs qui permettent de les atteindre

\begin{tabular}{|c|c|c|}
\hline $\begin{array}{l}\text { Catégories et identifiants } \\
\text { des classes }\end{array}$ & $\begin{array}{c}\text { Organisateurs sur lesquels les enseignants s'appuient pour } \\
\text { conduire leurs séances de lecture collectives }\end{array}$ & Buts inférés \\
\hline $\mathrm{N}^{0} 1$ - Classe 48 & $\begin{array}{l}\text { Lecture à haute voix } \\
\text { Compréhension qui précède la découverte de texte }\end{array}$ & Oraliser le texte \\
\hline No 2 - Classe 20 & $\begin{array}{l}\text { Reconnaissance des mots écrits } \\
\text { Compréhension qui précède la découverte de texte }\end{array}$ & Oraliser le texte \\
\hline No 3 - Classe 113 & Compréhension en cours de lecture & Comprendre des phrases \\
\hline No 6 - Classe 112 & $\begin{array}{l}\text { Compréhension du texte lu par l'adulte } \\
\text { Déchiffrage en contexte }\end{array}$ & $\begin{array}{l}\text { Comprendre un texte/ora- } \\
\text { liser une phrase }\end{array}$ \\
\hline No 4 - Classe 89 & Déchiffrage en contexte & Comprendre le texte \\
\hline No 5 - Classe 60 & $\begin{array}{l}\text { Déchiffrage en contexte et mise en relation des graphèmes et des } \\
\text { phonèmes }\end{array}$ & Oraliser un texte \\
\hline
\end{tabular}


giant le recours au contexte, lecture linéaire du texte à haute voix. Dix ans plus tard, le tableau que nous dressons est très différent et conforte les observations que nous avions faites au cours d'études antérieures (Goigoux, Riou et Serres, 2015; Riou, 2013).

Sur les 36 enseignants dont nous avons analysé les pratiques, 15 ne sollicitent ni description ni commentaire des illustrations et 8 s'appuient quasiexclusivement sur le déchiffrage pour identifier les mots écrits. En outre, la quasi-totalité des enseignants (33 sur 36) propose des tâches de déchiffrage en contexte et aucun ne joue aux devinettes contrairement à ce que laissent entendre les discours alarmistes sur l'état de l'école (Garcia et Oller, 2015). Le mode de parcours du texte est très souvent linéaire mais la lecture à haute voix, entrecoupée de tâches de déchiffrage, de reconnaissance de mots entiers, de lexique ou de compréhension, s'effectue rarement sans interruption. Bref, les modèles didactiques des séances de lecture collectives sont plus nombreux et variés que ne l'indiquait en 2005 le rapport conjoint de l'IGEN et de l'ONL, et il faut probablement y voir le signe d'une évolution des pratiques de classe.

Certaines de nos observations confortent cependant quelques éléments figurant dans ce rapport. Comme en 2005, le travail sur l'intégration syntaxique, c'est-à-dire sur l'ordre des mots de la phrase, l'enchainement propositionnel ou la cohésion anaphorique, est absent des séances de lecture collectives que nous avons analysées. Les enseignants sollicitent de la part des élèves des microprocessus, plus rarement des macroprocessus mais presque jamais des processus d'intégration. Autrement dit, les tâches qui incitent les élèves à construire progressivement la signification du texte à partir des informations contenues dans les phrases sont quasi absentes des scénarios didactiques. Le résultat le plus significatif, et le plus paradoxal, est donc la très faible place accordée aux tâches aboutissant à la compréhension de l'écrit (C-in) dans ce type de séances. Les tâches touchant la compréhension participent plus à la construction d'une motivation au décodage qu'au produit de cette activité. Notre étude met en lumière la difficulté des enseignants à articuler les traitements de bas niveaux et la construction de significations. Il semblerait donc que le travail sur le code et le sens soit difficile à concilier dans une même séance. L'enseignante de la classe 89 (catégorie $n^{0} 4$ ), qui parvient le mieux à le faire, émet elle-même des doutes à ce sujet en indiquant à ses élèves qu'elle a conscience de l'attention prolongée qu'elle leur demande. Nos résultats vont donc dans le sens des observations faites par Gérard Sensevy $(2007$; 2009) concernant la faible quantité d'interactions didactiques portant sur les interprétations plausibles d'un texte. En effet, le temps passé à résoudre des tâches qui concourent à l'élaboration de significations représente moins de $7 \%$ de la durée totale des séances. Au début des années 2000, Roland Goigoux (2004) avait évalué à un quart du temps du lire-écrire la proportion consacrée à la compréhension. Au cours de l'année scolaire 20132014, les enseignants expérimentés de cours préparatoire y consacrent seulement $16 \%$ de leur temps en moyenne (cf. annexe 2) et privilégient les tâches individuelles de compréhension écrite (Bishop, Cèbe et Piquée, 2015). Par ailleurs, comme l'avait noté Catherine Tauveron (2007) à propos de l'émission d'hypothèses, les enseignants ne font pas le même usage des tâches de même nature. La reconnaissance de mots entiers, par exemple, peut constituer le point de départ d'une séance de lecture collective, suivre l'identification de chacune des phrases du texte ou être proposée après que le texte ait été lu, passant ainsi du statut d'aide au décodage à celui de mémorisation orthographique des mots précédemment découverts.

L'analyse des séances de lecture collectives met en évidence les deux principaux buts que les enseignants poursuivent, à savoir l'oralisation et la compréhension. Ces buts correspondent à deux des objectifs identifiés par François Simon (2004), le troisième étant l'articulation entre « traitement de l'écrit » et « compréhension des unités lues ». Nous aboutissons donc à un résultat un peu différent dans la mesure où le traitement de l'écrit apparait davantage comme un moyen d'atteindre son but que comme un but en soi. En effet, l'oralisation peut s'effectuer sans qu'aucune tâche ne porte sur le traitement d'unités linguistiques de niveau lexical ou infralexical mais elle peut également prendre appui sur la reconnaissance orthographique de mots entiers ou mobiliser des tâches de déchiffrage avec ou sans explicitation des correspondances entre les graphèmes et les phonèmes. Autrement dit, les enseignants qui visent l'oralisation du texte choisissent majoritairement des tâches de lecture à haute voix, de reconnaissance de mots écrits ou de déchiffrage avec ou sans explicitation des procédures. La résolution de tâches de compréhension s'appuie toujours sur l'un de ces quatre modes d'oralisation. L'oralisation apparait donc comme un 
moment charnière : elle peut représenter la finalité de la tâche de lecture ou constituer un préalable au travail de compréhension. Il est également possible d'observer des lectures en deux temps, la première étape consistant à déchiffrer le texte, la seconde à le comprendre.

Les scénarios didactiques que nous avons décrits et analysés sont typiques des 36 séances de lecture collectives de notre étude et renvoient à six pratiques d'enseignement très différentes. Les représentations graphiques de ces scénarios nous ont permis de mettre en évidence les unités linguistiques sur lesquelles portent les traitements cognitifs des élèves, de rendre compte de la dynamique des séances, de repérer des épisodes structurant l'activité des enseignants, et par conséquent de donner une image extrêmement précise du déroulement de leur activité. En centrant notre attention sur les proportions de durées d'enseignement des différents types de tâches qui composent les séances de lecture collectives, nous avons réinterrogé la fonction de ces séances et leur rôle au sein d'autres composantes de l'enseignement de la lecture.

Les scénarios prototypiques que nous avons identifiés sont par construction fidèles à la situation de lecture collective, une situation de référence de l'enseignement du lire-écrire, et peuvent donc être utilisés en formation (Durand, 2009). Ils peuvent être présentés aux enseignants comme une sorte de miroir de la diversité de leurs pratiques et servir de point de départ à une élucidation des principes qui sous-tendent leurs choix. Il serait alors nécessaire de mobiliser les principaux organisateurs de l'activité d'enseignement afin d'apprendre aux formés à mieux connaitre les situations de travail auxquelles ils sont régulièrement confrontés, à comprendre « comment elles interagissent sur leur propre action, et comment ils peuvent agir sur elles » (Mayen, Métral et Tourmen, 2010).

\section{NOTES}

1. La séance didactique est définie comme une unité repérable dans l'emploi du temps quotidien des enseignants.

2. Les termes « déchiffrage " et " décodage » sont souvent employés indifféremment pour désigner le traitement d'une suite de signes écrits en vue de leur sonorisation ou de l'accès au sens du message. Dans cet article, nous utiliserons le terme « déchiffrage » lorsque la tâche à résoudre consistera à sonoriser un mot et le terme « décodage » lorsqu'il s'agira de désigner l'ensemble des traitements écrits qui visent l'accès au sens du texte.

3. PIREF : Programme incitatif de recherche en éducation et formation.

4. Les tâches de lecture à haute voix proposées par l'enseignant conduisent à des réalisations diverses selon que les élèves qui les résolvent rencontrent ou non des difficultés d'identification des mots écrits. Ainsi, les phrases peuvent être lues dans leur intégralité sans interruption mais cette lecture peut être entrecoupée par la résolution de tâches de différentes natures telles que la mise en relation des graphèmes et des phonèmes, le travail sur les syllabes ou la combinatoire, l'explicitation du sens d'un mot, etc.

5. Nous appelons «temps morts» les temps qui ne sont pas consacrés à l'enseignement de la lecture ou de l'écriture mais qui s'intercalent dans une séance didactique

6. $\sigma$ signifie écart-type

7. Nous n'avons pas pris en compte l'étude de la correspondance graphophonémique $\mathrm{p} / \mathrm{p}$ / étudiée la même semaine que le texte, par souci d'équité avec les autres classes.

\section{RÉFÉRENCES}

Bishop, M.-F., Cèbe, S. et Piquée, C. (2015). L'enseignement de la compréhension dans les classes de CP aujourd'hui : temps consacré et choix didactiques. Repères, 52, 15-38.

Durand, M. (2009). Analyse du travail dans une visée de formation : cadres théoriques, méthodes et conceptions. Dans J.-M. Barbier, E. Bourgeois, G. Chapelle et J. C. Ruano-Borbalan (dir), Encyclopédie de la formation (p. 827-856). Paris : Presses universitaires de France.

Fijalkow, E. et Fijalkow, J. (1994). Enseigner à lire-écrire au CP : état des lieux. Revue française de pédagogie, 107, 63-79.

Garcia, S. et Oller, A.-C. (2015). Réapprendre à lire : De la querelle des méthodes à l'action pédagogique. Paris : Éditions du Seuil.

Giasson, J. (1990). La compréhension en lecture. Québec, Canada : Gaëtan Morin.

Goigoux, R. (2002). Analyser l'activité d'enseignement de la lecture : une monographie. Revue française de pédagogie, 138, 125-134.

Goigoux, R. (2004). Méthodes et pratiques d'enseignement de la lecture. Formation et pratiques d'enseignement en questions, 1, 37-56.

Goigoux, R. (2007). Un modèle d'analyse de l'activité des enseignants. Éducation $\&$ didactique, 1(3), 47-69.

Goigoux, R. (dir.) (2016). Étude de l'influence des pratiques d'enseignement de la lecture et de l'écriture sur la qualité 
des premiers apprentissages (Rapport de recherche). [Récupéré du site de l'institut français de l'éducation : http://ife.ens-lyon.fr/ife/recherche/lire-ecrire/rapport/ rapport].

Goigoux, R., Riou, J. et Serres, G. (2015). La régulation de l'action des enseignants. Travail et Apprentissages, $15,66-83$.

Inserm (2007). Dyslexie, dysorthographie, dyscalculie : bilan des données scientifiques (Expertise collective). Paris : Les éditions Inserm. [Récupéré de http://ipubliinserm.inist.fr/handle/10608/73].

Inspection générale de l'éducation nationale et Observatoire national de la lecture (2005). L'apprentissage de la lecture à l'école primaire. [Récupéré du site du ministère de l'éducation nationale : http://media.education. gouv.fr/file/96/0/5960.pdf] (PDF).

Jarlégan, A. (2015). Estimer l'ampleur des effets-classes avant d'identifier les processus susceptibles de les produire. Document de travail interne à la recherche Lire et Écrire.

Jaubert, M. et Rebière, M. et avec la collaboration de Bernié, J.P. (2010). Forme scolaire et genres professionnels : une question pour la formation. Un exemple : la « lecture-découverte d'un texte narratif » au CP. Travail et formation en éducation, 5, mis en ligne le 17 juin 2010, consulté le 14 mars 2018.

Leplat, J. (1997). Regards sur l'activité en situation de travail. Contribution à la psychologie ergonomique. Paris: Presses universitaires de France.

Maurice, J.-J. (1996a). Une connaissance de l'élève dépendante des contraintes de l'action. Revue française de pédagogie, 114, 85-96.

Maurice, J.-J. (1996b). Problèmes multiplicatifs : l'expérience de l'enseignant, l'action effective de l'élève. Recherche en Didactique des Mathématiques, 16(3), 323-348.

Maurice, J.-J. (1996c). Modélisation du savoir-faire de l'enseignant expérimenté : adaptation aux contraintes, anticipation, négociation, pilotage de la classe par les tâches scolaires (thèse de doctorat non publié). Université Pierre-Mendès-France, Grenoble.

Maurice, J.-J. (2005). Les tâches proposées aux élèves : des instruments de pilotage de la classe. Former des enseignants-professionnels, savoirs $\varepsilon$ compétences. Communication présentée au $5^{\mathrm{e}}$ colloque international IUFM Pays de la Loire, Nantes.

Mayen, P., Métral, J.-F, et Tourmen, C. (2010). Les situations de travail. Références pour les référentiels. Recherche et formation, 64, 31-46 [http://dx.doi. org/10.4000/rechercheformation.191].

Ministère de l'Éducation nationale, de l'enseignement supérieur et de la recherche (2015). Programmes pour les cycles 2, 3, 4. Bulletin officiel spécial $n^{\circ} 11$ du 26 novembre 2015.

National Institute of Child Health and Human Development (NICHD), National Institutes of Health (NIH) (2000). National Reading Panel (NRP). Teaching Children to Read: an Evidence-Based Assessment of the Scientific
Research Literature on Reading and Its Implications for Reading Instruction: Reports of the Subgroups. Washington, DC: U.S. Government Printing Office.

Pastré, P. (1999). La conceptualisation dans l'action. Bilan et nouvelles perspectives. Éducation permanente, 139, 13-35.

Riou, J. (2013). Quels sont les indicateurs qui permettent à l'enseignant de réguler son activité ? (La situation de découverte de texte au cours préparatoire). Mémoire de Master 2 sous la direction de Guillaume Serres. Master international francophone des métiers de la formation, Université Blaise Pascal, Clermont-Ferrand.

Simon, F. (2004). Les pratiques d'enseignement-apprentissage de la lecture au cours préparatoire : positionnement des activités de compréhension et de traitement de l'écrit. Interactions entre processus d'enseignement et activité des élèves dans une situation de découverte de texte (thèse de doctorat non publiée). Université de Nantes.

Simon, F. (2007). Gérer l'hétérogénéité des savoirs des élèves dans une activité de lecture collective de texte au cours préparatoire. Repères, 36, 77-99 [http:// dx.doi.org/10.4000/reperes.468].

Sensevy, G. (2007). Caractérisation des pratiques d'enseignement et détermination de leur efficacité. La lecture et les mathématiques au cours préparatoire. Rapport de Recherche pour le Programme Incitatif de Recherche en éducation et Formation (PIREF). Paris : Ministère de la jeunesse, de l'éducation et de la recherche.

Sensevy, G. (2009). Étude d'un enseignement de la lecture au cours préparatoire : esquisse d'articulation de divers types d'analyse. Revue française de pédagogie, 168, p. 39-58 [http://dx.doi.org/10.4000/rfp.1734].

Tauveron, C. (2007). Lecture d'un même texte au même moment dans trois cours préparatoires différents. Analyse comparative. Repères, 36, 121-147.

Vergnaud, G. (2007). Apprentissage et développement : apprendre, se former et agir Représentation et activité : deux concepts étroitement associés. Recherches en Éducation, 4, 9-23.

Vidal-Gomel, C. et Rogalski, J. (2007). La conceptualisation et la place des concepts pragmatiques dans l'activité professionnelle et le développement des compétences. Activités, 4(1) [http://dx.doi.org/10.4000/ activites.1401]. 


\section{ANNEXE 1 : TYPOLOGIE DES TÂCHES DE L'ÉTUdE LIRE ET ÉCRIRE (GOIGOUX [DIR.], 2016)}

$\varnothing:$ Temps mort

MD : Mémoire didactique

MD1. Rappeler ou réviser un apprentissage antérieur MD2. Récapituler un nouvel apprentissage

PG : Phono - graphie

PG1. Étudier les phonèmes (sans écrit)

PG2. Étudier les syllabes orales ou d'autres unités de taille supérieure au phonème (rime) (sans écrit)

G3. Étudier les lettres (noms; différentes écritures ; sans valeur sonore)

PG4. Étudier les correspondances entre phonèmes et graphèmes (CGP)

PG5. Étudier la combinatoire ou travailler sur les syllabes

\section{L : Lecture}

L1. Lire silencieusement [M P T]*

L2. Reconnaitre un mot entier

L3. Déchiffrer un mot

L4. Lire à haute voix [P T]

L5. Écouter la maitresse/le maitre lire à haute voix

$[\mathrm{P} \mathrm{T}]$

EL : Étude de la langue

EL1. Lexique (sens et construction des mots)

EL2. Syntaxe (ordre des mots et valeur des mots outils)

EL3. Morphologie (orthographe des mots, chaine des accords et désinences verbales)

É : Écriture

E1. Calligraphier [L S M]

E2. Copier (avec modèle) [L S M P T]

E3. Copier après disparition du modèle [L S M P T] (copie différée)

E4. Écrire sous la dictée [L S M P T] (Le maitre décide des unités à écrire)

E5. Produire en combinant des unités linguistiques déjà imprimées [S M P T] (coder l'unité à produire)

E6. Produire en dictant à autrui [L S M P T]

E7. Produire en encodant soi-même [S M P T] (Les élèves choisissent)

E8. Définir, planifier ou organiser la tâche d'écriture (enjeu, destinataire, contenu, plan...)

E9. Revenir sur l'écrit produit : le commenter, le corriger, le réviser, l'améliorer.

$C$ : Compréhension

C1. Définir ou expliciter une intention de lecture

C2. Anticiper, formuler ou vérifier des hypothèses

C3. Décrire, commenter une illustration
C4. Expliquer ou reformuler le sens ou évoquer une représentation mentale [P T]

C5. Produire un rappel de récit (complet ou partiel) ou un rappel de texte explicatif ou de consigne

C6. Rendre explicite une information implicite

C7. Proposer, débattre ou négocier une interprétation/des interprétations

C8. Réaliser une tâche écrite impliquant la compréhension (explicite et/ou implicite) [M P T]

C9. Corriger une tâche écrite portant sur la compréhension [M P T]

AUTR: Autre type de tâche (préciser laquelle dans la rubrique « Commentaire »)

${ }^{*} \mathrm{~L}=$ Lettre $; \mathrm{S}=$ Syllabe $; \mathrm{M}=$ Mot $; \mathrm{P}=$ Phrase $;$ $\mathrm{T}=$ Texte 


\section{ANNEXE 2}

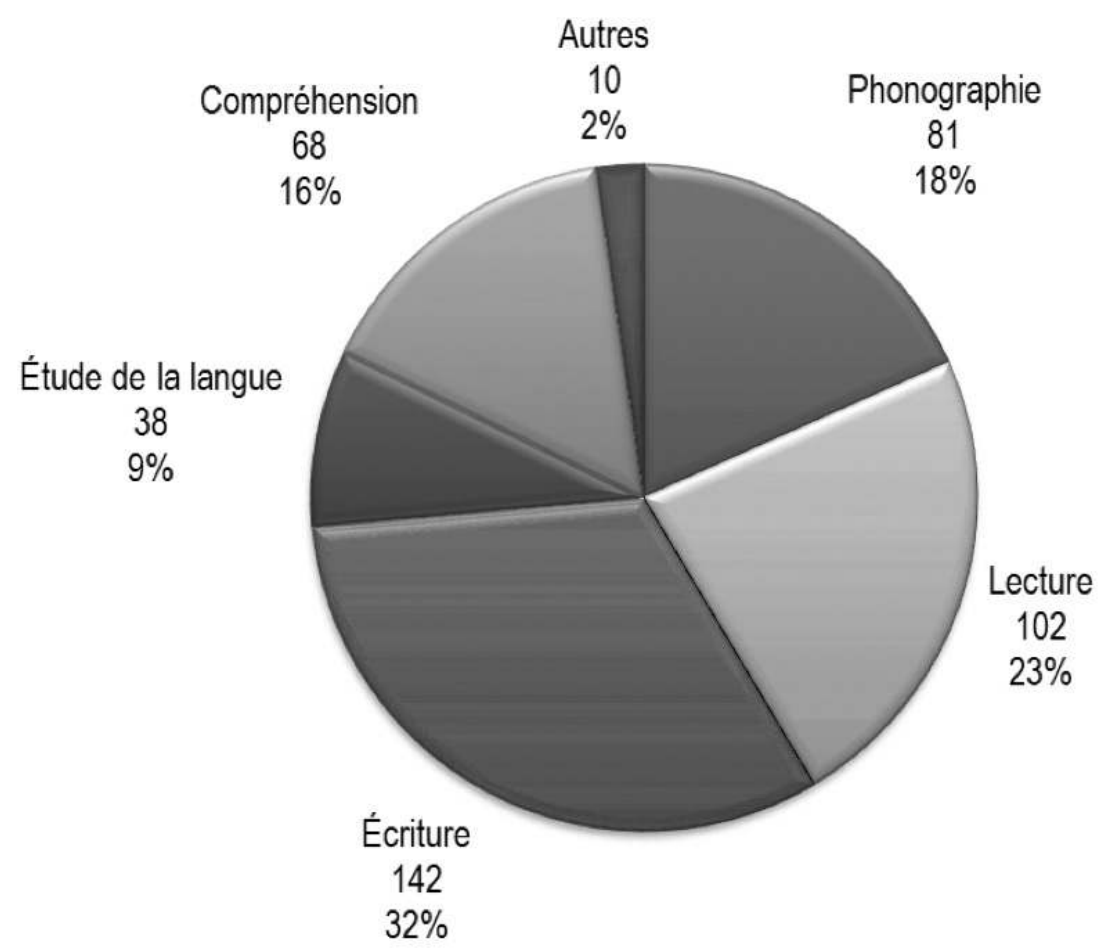

Durées hebdomadaires moyennes (en minutes) et proportions des grands ensembles de tâches de lecture et d'écriture relevées dans 131 classes de cours préparatoire 